\title{
Safety and effectiveness of early chemical deep venous thrombosis prophylaxis after spinal cord injury: pilot prospective data
}

\author{
Anthony M. DiGiorgio, DO, MHA, ${ }^{1,5}$ Rachel Tsolinas, BA, ${ }^{2}$ Mohanad Alazzeh, BS, ${ }^{3}$ \\ Jenny Haefeli, PhD, ${ }^{2}$ Jason F. Talbott, MD, PhD, ${ }^{4}$ Adam R. Ferguson, $\mathrm{PhD},{ }^{2}$ \\ Jacqueline C. Bresnahan, PhD, ${ }^{2}$ Michael S. Beattie, PhD, ${ }^{2}$ Geoffrey T. Manley, MD, PhD, ${ }^{5}$ \\ William D. Whetstone, MD, ${ }^{6}$ Praveen V. Mummaneni, MD, ${ }^{5}$ and Sanjay S. Dhall, MD
}

\begin{abstract}
${ }^{1}$ Department of Neurosurgery, Louisiana State University Health Sciences Center, New Orleans, Louisiana; ${ }^{2}$ UCSF Brain and Spinal Injury Center, and ${ }^{4}$ Department of Radiology and Biomedical Imaging, Zuckerberg San Francisco General Hospital, San Francisco; ${ }^{3}$ David Geffen School of Medicine, University of California, Los Angeles; and Departments of ${ }^{5}$ Neurological Surgery and ${ }^{6}$ Emergency Medicine, University of California, San Francisco, California
\end{abstract}

OBJECTIVE Spinal cord injuries (SCIs) occur in approximately 17,000 people in the US each year. The average length of hospital stay is 11 days, and deep venous thrombosis (DVT) rates as high as $65 \%$ are reported in these patients. There is no consensus on the appropriate timing of chemical DVT prophylaxis for this critically injured patient cohort. The object of this study was to determine if low-molecular-weight heparin (LMWH) was safe and effective if given within 24 hours of SCl.

METHODS The Transforming Research and Clinical Knowledge in SCls study is a prospective observational study conducted by the UCSF Brain and Spinal Injury Center. Protocol at this center includes administration of LMWH within 24 hours of SCI. Data were retrospectively reviewed to determine DVT rate, pulmonary embolism (PE) rate, and hemorrhagic complications

RESULTS Forty-nine patients were enrolled in the study. There were 3 DVTs (6.1\%), 2 PEs (4.1\%), and no hemorrhagic complications. Regression modeling did not find an association between DVT and/or PE and age, American Spinal Injury Association grade, sex, race, or having undergone a neurosurgical procedure.

CONCLUSIONS A standardized protocol in which LMWH is given to patients with SCl within 24 hours of injury is effective in keeping venous thromboembolism at the lower end of the reported range, and is safe, with a zero rate of adverse bleeding events.

https://thejns.org/doi/abs/10.3171/2017.8.FOCUS17437

KEY WORDS spinal cord injury; DVT prophylaxis; low-molecular-weight heparin; spine trauma; thromboembolic events

$\mathrm{S}$ PINAL cord injuries (SCIs) in the US have been on the rise, with an estimated yearly incidence of 17,000 cases. ${ }^{13}$ Spinal cord injuries are most prevalent in middle-aged white males, with motor vehicle collisions as the most common cause. ${ }^{8,13}$ Approximately 243,000347,000 people in the US live with an SCI, with $30 \%$ hospitalized at least once a year after discharge. The initial length of acute hospitalization for SCIs is 11 days, with 35 days of rehabilitation..$^{10,13}$

Deep venous thrombosis (DVT) and pulmonary embo- lism (PE) in SCI are common. Together these events are referred to as venous thromboembolism (VTE). Reported DVT rates in the literature are variable, as high as $65 \%$ in some studies. ${ }^{4,12}$ The PE rates also have a variable reported range from $0 \%$ to $18 \%{ }^{4,6,11,12}$ In a cohort study of the shortterm and long-term risk of a VTE in 94 patients with SCI, it was found that most VTE complications occur within 3 months of the SCI. ${ }^{5}$

The 2013 SCI guidelines were published by the American Association of Neurological Surgeons/Congress of

ABBREVIATIONS AANS/CNS = American Association of Neurological Surgeons/Congress of Neurological Surgeons; DVT $=$ deep venous thrombosis; $L$ LMWH $=$ low-molecular-weight heparin; $\mathrm{PE}=$ pulmonary embolism; $\mathrm{SCl}=$ spinal cord injury; TRACK = Transforming Research and Clinical Knowledge; VTE = venous thromboembolism. SUBMITTED June 30, 2017. ACCEPTED August 4, 2017. INCLUDE WHEN CITING DOI: 10.3171/2017.8.FOCUS17437. 
Neurological Surgeons (AANS/CNS) Joint Section on Disorders of the Spine and Peripheral Nerves. These guidelines give a Class I recommendation for low-molecular-weight heparin (LMWH) use for VTE prophylaxis and a Class II recommendation for starting within 72 hours of injury. ${ }^{3}$

Transforming Research and Clinical Knowledge in SCIs (TRACK SCI) is a prospective observational study based at the University of California, San Francisco. It introduced a standardized SCI treatment protocol and prospective data collection. The trauma protocol includes administration of LMWH (enoxaparin) within 24 hours of injury. The aim of the present study is to review the TRACK SCI data and determine if there is a reduction in VTE over established rates, as well as to evaluate the safety of early LMWH administration through assessment of adverse hemorrhagic events.

\section{Methods}

The TRACK SCI is a prospective observational study conducted by the UCSF Brain and Spinal Injury Center. This study recruited patients at Zuckerberg San Francisco General Hospital's Level I Trauma Center. Institutional review board approval was obtained at this site for all study procedures.

Patients were enrolled from May 2015 through March 2017. All English-speaking and non-English-speaking patients who presented to the emergency department and who were diagnosed with a traumatic SCI, whether blunt or penetrating, were initially eligible for the study. Patients who were younger than 18 years, in custody, prisoners, pregnant, or on medically evaluated psychiatric hold were excluded from the study. Informed consent was obtained for all patients.

The standard protocol for these patients involves initiating chemical DVT prophylaxis with LMWH (40 mg subcutaneous enoxaparin administered daily) within 24 hours of injury. Patients with SCI who undergo operation are taken for surgery within 24 hours of injury, and LMWH is withheld for 24 hours after surgery. Due to the nature of referral patterns in San Francisco, Zuckerberg San Francisco General Hospital does not receive trauma patients transferred from other institutions.

Data from the TRACK SCI database were reviewed. The DVT and PE rates were recorded along with any hemorrhagic complications. Patients did not undergo routine screening. Duplex ultrasound was performed if there was clinical suspicion for DVT or PE, and PE was confirmed with CT angiography. Multivariate regression modeling was performed using IBM SPSS (IBM Corp.).

\section{Results}

Of the 49 patients enrolled, $32(65.3 \%)$ were male. The average age was 53.5 years, with a range of $18-49$ years. The largest group of patients was Caucasian $(38.8 \%, \mathrm{n}=$ 19); followed by Asian $(24.5 \%, \mathrm{n}=12)$; African American $(18.4 \%, \mathrm{n}=9)$; and Hispanic $(16.3 \%, \mathrm{n}=8)$, with only 1 patient who was "other" or declined to state (2\%). The mean time from injury to emergency department arrival averaged 17 minutes. Complete SCIs were present in 9 patients
(18.4\%), and $40(81.6 \%)$ underwent a spinal surgery. Seven patients (14.3\%) had additional nonneurosurgical injuries. The average ICU stay was 8.6 days, and the average hospital length of stay was 15.9 days. Discharge dispositions were rehabilitation $(36.7 \%, \mathrm{n}=18)$; nursing facility $(20.4 \%$, $\mathrm{n}=10)$; home $(14.3 \%, \mathrm{n}=7)$; and another acute care facility $(20.4 \%, \mathrm{n}=10)$. A total of 4 patients $(8.2 \%)$ died during their hospital stay. Of those who survived, only 1 patient had a neurological decline from presentation, which was later attributed to a cerebrovascular accident. See Table 1 for a summary of results.

There were 3 DVTs (6.1\%), 2 PEs (4.1\%), and no hemorrhagic complications (Table 2). Regression modeling did not find an association between DVT or PE and age, American Spinal Injury Association grade, sex, race, or having undergone a neurosurgical procedure $(p>0.05$ for all variables).

\section{Discussion}

The existing literature does not provide clear data on when to start chemical DVT prophylaxis, and there is a great deal of caution among spine surgeons about the potential for hemorrhagic complications. In the current published literature, there is only 1 study that specifically examines the timing of chemical DVT prophylaxis. A prospective trial by Aito et al. compared patients presenting with acute SCI who received LMWH on hospital admission to their rehabilitation center. The admission window was between 72 hours of injury or after 8 days. Within the 72-hour cohort, $2 \%$ of patients developed a DVT on routine ultrasonography versus $26 \%$ of patients in the other cohort. ${ }^{1}$ There were no adverse bleeding events in either group.

Other studies have specified time to chemical DVT prophylaxis, but have not directly compared differing times. A randomized controlled trial by the SCI investigators compared subcutaneous heparin to LMWH administered within 72 hours of SCI. They found a DVT rate of $63.3 \%$ in the subcutaneous heparin group versus $65.5 \%$ with LMWH, and a PE rate of $18.4 \%$ versus $5.2 \%$ for subcutaneous heparin versus LMWH, respectively. ${ }^{12}$ A retrospective review by Harris et al. looked at LMWH started at admission after SCI. Not all of the patients in their group underwent surgery within 24 hours of injury, so for them the LMWH was started on admission, withheld 24 hours prior to the operation, and then restarted 24 hours after. There was no evidence of DVT; however, there were 3 episodes of bleeding complications that were attributed to the therapy. ${ }^{9}$

The recommended range of time to wait before starting chemical DVT prophylaxis varies widely. Ploumis et al. recommend starting LMWH within 2 weeks, ${ }^{14}$ whereas Christie et al. recommend 72 hours. $^{2}$ The AANS/CNS Joint Section on Trauma and Neurocritical Care suggests starting LMWH for DVT prophylaxis within 72 hours. ${ }^{3}$

There is, similarly, a wide variation of DVT rates in the literature, due to a multitude of prophylaxis and screening methods. For example, Gündüz et al. found a 53.3\% DVT rate with subcutaneous heparin prophylaxis and routine venography. ${ }^{7}$ A meta-analysis by Furlan and Fehlings 
TABLE 1. Demographics of the 49 patients with SCI prospectively tracked in this study

\begin{tabular}{lc}
\hline \multicolumn{1}{c}{ Factor } & Value \\
\hline Male sex & $32(65.3)$ \\
\hline Race & $19(38.8)$ \\
\hline Caucasian & $12(24.5)$ \\
\hline Asian & $9(18.4)$ \\
\hline African American & $8(16.3)$ \\
\hline Hispanic & $1(2)$ \\
\hline Other/declined to state & $9(18.4)$ \\
\hline Complete SCl on admission & $40(81.6)$ \\
\hline Underwent spinal procedure & $7(14.3)$ \\
\hline Additional nonneurosurgical injuries & $8.59 \pm 8.02$ \\
\hline Mean ICU stay in days, \pm SD & $15.88 \pm 15.47$ \\
\hline Mean hospital length of stay in days, \pm SD & $18(36.7)$ \\
\hline Discharge disposition & $10(20.4)$ \\
\hline Rehabilitation & $7(14.3)$ \\
\hline Nursing facility & $10(20.4)$ \\
\hline Home & $4(8.2)$ \\
\hline Acute care
\end{tabular}

Values are expressed as the number (\%) of patients unless otherwise noted.

found rates from $6 \%$ to $50 \%$, and also concluded that routine DVT screening is not recommended. ${ }^{4}$

Few studies have examined the adverse bleeding rates after LMWH administration. The SCI investigators looked at 230 patients receiving enoxaparin within 72 hours of SCI. They had a $2.6 \%$ rate of major bleeding complications, $14.8 \%$ risk of minor bleeding complications, and, as mentioned above, DVT and PE rates of $65.5 \%$ and $5.2 \%$, respectively. ${ }^{12}$

With our aggressive LMWH administration, the data show a $6.1 \%$ DVT and a $4.1 \%$ PE rate, with an absence of bleeding events. Although our thromboembolic event rate is higher than in the retrospective study by Harris et al., ${ }^{9}$ it is on the lower end of the commonly reported DVT rates in the literature. Our study's main significance comes with the absence of adverse bleeding events. This suggests that LMWH is safe if given within 24 hours of injury, as is done with our standard protocol.

The TRACK SCI is a prospectively collected SCI database. This makes our study unique in that it is based on prospectively collected data with a defined chemical DVT prophylaxis protocol. Although this is a retrospective review of the data, the standardized, prospective nature of the TRACK SCI data collection helps to minimize many

TABLE 2. Thromboembolic events and hemorrhagic complications from aggressive LMWH therapy

\begin{tabular}{lc}
\hline Thromboembolic Event & No. (\%) \\
\hline DVT & $3(6.1)$ \\
\hline PE & $2(4.1)$ \\
\hline Hemorrhagic complications & 0 \\
\hline
\end{tabular}

of the inherent biases in retrospective chart reviews-notably selection bias. A limitation of this study is the relatively smaller sample size when compared with the retrospective reviews cited here.

Our data reiterate the prospective data found by Aito et al., that early LMWH is safe and can lower DVT rates substantially when given within 72 hours of SCI. ${ }^{1}$ The other prospective study, by the SCI investigators, had a similar PE rate to ours; however, their routine DVT screening yielded a much higher DVT rate. ${ }^{12}$ Furthermore, our early pilot data suggest that the common concern about hemorrhagic complications from prophylactic doses of enoxaparin may not be as substantial as once believed.

\section{Conclusions}

A standardized protocol in which LMWH is given to patients with SCI within 24 hours of injury is effective in keeping VTE at the lower end of the reported range, and is safe, with a zero rate of adverse bleeding events.

\section{Acknowledgments}

Partial support was provided by the Department of Defense Congressionally Directed Medical Research Program (CDMRP) award SC120259 (M.S.B., G.T.M., J.C.B., W.D.W., A.R.F.) and the Craig H. Nielsen Foundation (J.H.).

\section{References}

1. Aito S, Pieri A, D’Andrea M, Marcelli F, Cominelli E: Primary prevention of deep venous thrombosis and pulmonary embolism in acute spinal cord injured patients. Spinal Cord 40:300-303, 2002

2. Christie S, Thibault-Halman G, Casha S: Acute pharmacological DVT prophylaxis after spinal cord injury. J Neurotrauma 28:1509-1514, 2011

3. Dhall SS, Hadley MN, Aarabi B, Gelb DE, Hurlbert RJ, Rozzelle CJ, et al: Deep venous thrombosis and thromboembolism in patients with cervical spinal cord injuries. Neurosurgery 72 (Suppl 2):244-254, 2013

4. Furlan JC, Fehlings MG: Role of screening tests for deep venous thrombosis in asymptomatic adults with acute spinal cord injury: an evidence-based analysis. Spine (Phila Pa 1976) 32:1908-1916, 2007

5. Giorgi Pierfranceschi M, Donadini MP, Dentali F, Ageno W, Marazzi M, Bocchi R, et al: The short- and long-term risk of venous thromboembolism in patients with acute spinal cord injury: a prospective cohort study. Thromb Haemost 109:34-38, 2013

6. Green D, Chen D, Chmiel JS, Olsen NK, Berkowitz M, Novick A, et al: Prevention of thromboembolism in spinal cord injury: role of low molecular weight heparin. Arch Phys Med Rehabil 75:290-292, 1994

7. Gündüz S, Oğur E, Möhür H, Somuncu I, Açjksöz E, Ustünsöz B: Deep vein thrombosis in spinal cord injured patients. Paraplegia 31:606-610, 1993

8. Hagen EM, Rekand T, Gilhus NE, Grønning M: Traumatic spinal cord injuries-incidence, mechanisms and course. Tidsskr Nor Laegeforen 132:831-837, 2012

9. Harris S, Chen D, Green D: Enoxaparin for thromboembolism prophylaxis in spinal injury: preliminary report on experience with 105 patients. Am J Phys Med Rehabil 75:326-327, 1996

10. Jain NB, Ayers GD, Peterson EN, Harris MB, Morse L, O'Connor KC, et al: Traumatic spinal cord injury in the United States, 1993-2012. JAMA 313:2236-2243, 2015 
11. Kulkarni JR, Burt AA, Tromans AT, Constable PD: Prophylactic low dose heparin anticoagulant therapy in patients with spinal cord injuries: a retrospective study. Paraplegia 30:169-172, 1992

12. Merlie G, Geerts W, Ginzburg E, Green D, Lambert R, Leduc B, et al: Prevention of venous thromboembolism in the acute treatment phase after spinal cord injury: a randomized, multicenter trial comparing low-dose heparin plus intermittent pneumatic compression with enoxaparin. J Trauma 54:1116-1126, 2003

13. National Spinal Cord Injury Statistical Center: Facts and Figures at a Glance. (https://www.nscisc.uab.edu/Public/ Facts\%202016.pdf) [Accessed September 15, 2017]

14. Ploumis A, Ponnappan RK, Maltenfort MG, Patel RX, Bessey JT, Albert TJ, et al: Thromboprophylaxis in patients with acute spinal injuries: an evidence-based analysis. J Bone Joint Surg Am 91:2568-2576, 2009

\section{Disclosures}

Dr. Dhall reports being a consultant for DePuy Spine. Dr. Mummaneni reports being a consultant for DePuy Spine and Stryker Spine; receiving honoraria from Globus and AOSpine; having direct stock ownership in Spinicity ISD; receiving royalties from DePuy Spine, Springer Publishing, and Thieme Publishing; and receiving support from ISSG for a non-study-related clinical or research effort that he oversees.

\section{Author Contributions}

Conception and design: DiGiorgio, Tsolinas, Haefeli, Talbott, Ferguson, Bresnahan, Beattie, Manley, Whetstone, Dhall. Acquisition of data: DiGiorgio, Tsolinas, Haefeli, Talbott, Ferguson, Bresnahan, Beattie, Manley, Whetstone, Dhall. Analysis and interpretation of data: DiGiorgio, Tsolinas, Dhall. Drafting the article: DiGiorgio, Tsolinas, Alazzeh, Dhall. Critically revising the article: DiGiorgio, Tsolinas, Alazzeh, Haefeli, Talbott, Ferguson, Bresnahan, Beattie, Manley, Whetstone, Dhall. Reviewed submitted version of manuscript: DiGiorgio, Tsolinas, Dhall. Approved the final version of the manuscript on behalf of all authors: DiGiorgio. Statistical analysis: DiGiorgio, Tsolinas, Dhall. Administrative/technical/material support: Tsolinas, Haefeli, Talbott, Ferguson, Bresnahan, Beattie, Manley, Whetstone, Mummaneni, Dhall. Study supervision: Manley, Mummaneni, Dhall.

\section{Correspondence}

Anthony M. DiGiorgio, Department of Neurosurgery, Louisiana State University Health Sciences Center, 2020 Gravier St., 7th Fl., New Orleans, LA 70112. email: adigi2@1suhsc.edu. 